\title{
Encoding visual stimuli with a population of Hodgkin-Huxley neurons
}

\author{
Aurel A Lazar ${ }^{*}$, Yiyin Zhou \\ From Nineteenth Annual Computational Neuroscience Meeting: CNS*2010 \\ San Antonio, TX, USA. 24-30 July 2010
}

In recent years the increasing availability of multi-electrode recordings has led to the application of neural decoding techniques to the recovery of complex stimuli such as natural scenes. A linear decoding algorithm was presented in [1] for the reconstruction of natural scenes with recognizable moving objects using recordings from a neural population of the cat's Lateral Geniculate Nucleus (LGN).

Most of the current models of encoding in the early visual system (retina, LGN, V1) consist of a linear receptive field followed by a non-linear spike generation mechanism. In [2] we considered a neural circuit architecture consisting of receptive fields in cascade with an equal number of spiking neural circuits. The neural circuits investigated were integrate-and-fire neurons and ON-OFF neurons with random thresholds and feedback. We demonstrated for the first time a decoding algorithm for natural scenes and shown its dependence on the noise level.

\section{Methods}

We investigate a neural encoding architecture for visual stimuli consisting of classical receptive fields (center surround or Gabor) in cascade with an ensemble of Hodgkin-Huxley neurons. Recovery of stimuli encoded with an ensemble of Hodgkin-Huxley neurons with known phase response curves was achieved based on the I/O equivalence between Hodgkin-Huxley neurons and Project-Integrate-and-Fire neurons in [3]. The ensemble of Hodgkin-Huxley neurons considered here is assumed to have unknown phase response curves [4]. We provide a visual stimulus reconstruction algorithm based on the spike times generated by the ensemble of HodgkinHuxley neurons and demonstrate its performance using natural video sequences (movies). Fig. 1 shows a sample

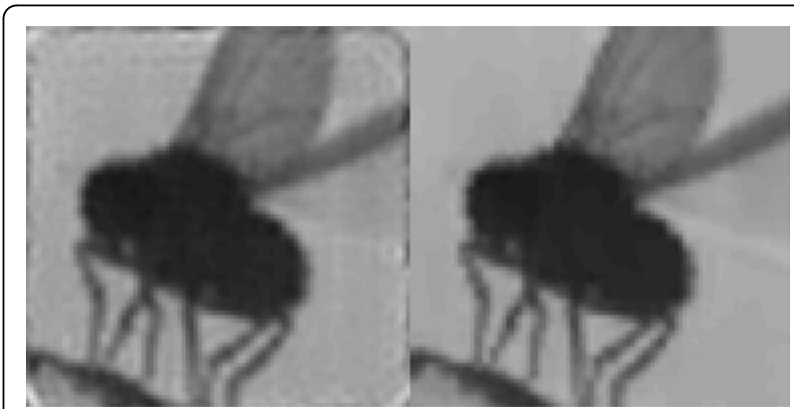

Figure 1

time instant (a frame of a movie) of the reconstructed (left) and the original (right) visual stimulus.

\section{Acknowledgements}

The work presented here was supported by AFOSR under grant number FA9550-09-1-0350.

\section{Published: 20 July 2010}

\section{References}

1. Stanley GB, Li FF, Dan Y: Reconstruction of Natural Scenes from Ensemble Responses in the Lateral Geniculate Nucleus. J Neurosci 1999, 19(18):8036-8042.

2. Lazar AA, Pnevmatikakis EA, Zhou Y: Encoding of Natural Scenes with Neural Circuits with Random Thresholds. BNET Technical Report \#06-09, Department of Electrical Engineering, Columbia University, New York, NY 2009.

3. Lazar AA: Population Encoding with Hodgkin-Huxley Neurons. IEEE Transactions on Information Theory 2010, 56(2), to appear.

4. Kim AJ, Lazar AA: Recovery of Stimuli Encoded with a Hodgkin-Huxley Neuron Using Conditional PRCs. In Phase Response Curves in Neuroscience Springer Nathan W. Schultheiss, Astrid Prinz, and Rob Butera 2010, to appear.

doi:10.1186/1471-2202-11-S1-P180

Cite this article as: Lazar and Zhou: Encoding visual stimuli with a population of Hodgkin-Huxley neurons. BMC Neuroscience 2010 11(Suppl 1):P180.

\footnotetext{
* Correspondence: aurel@ee.columbia.edu

Department of Electrical Engineering, Columbia University, New York, NY, USA
} 